H. TSUJI

KODAI MATH. J.

19 (1996), $137-143$

\title{
ON THE UNIVERSAL COVERING OF PROJECTIVE MANIFOLDS OF GENERAL TYPE
}

\author{
Hajime TSujI
}

\section{Introduction}

Around 1970, S. Kobayashi proposed the following conjecture ([3]).

Conjecture 1. Let $M$ be a compact Kähler manifold. Suppose that $M$ is measure hyperbolic. Then $M$ is of general type.

We note that a compact complex manifold $M$ of general type is always measure hyperbolic ([4, p. 9, Lemma 1$])$.

Recently M. Gromov introduced the notion of Kähler hyperbolicity and proved that Kähler hyperbolic manifolds are projective of general type ([2]). The main tools in the paper are Atiyah's $L^{2}$-index theorem and a Lefschetz type theorem. We note that Kähler hyperbolicity is a property of the universal covering manifold.

Although Gromov's theorem is a partial affirmative answer of Kobayashi's conjecture it seems to be hard to check that a given complex manifold is Kähler hyperbolic.

In this paper we shall give a partial affirmative answer for Kobayashi's conjecture for a compact quotient of a universal covering of a projective manifold of general type.

THEOREM 1. Let $X$ be a projective manifold of general type and let $\pi: D \rightarrow X$ be the universal covering of $X$. Then any compact unramified quotient of $D$ is of general type.

Remark 1. If the canonical bundle of $X$ is ample, $X$ carries a KählerEinstein metric $g_{E}$ of negative Ricci curvature by the solution of Calabi's conjecture $([1,7])$. By Yau's Schwarz lemma ([8]), $\pi^{*} g_{E}$ is invariant under the action of $\operatorname{Aut}(D)$. Hence every compact unramified quotient of $D$ carries a metric of strictly negative Ricci curvature. This implies that every compact quotient of $D$ has ample canonical bundle. In particular such a manifold is of general type.

Received October 27, 1994 ; revised June 27, 1995. 


\section{Proof of Theorem 1}

Let $X$ be a projective manifold of general type and let $\pi: D \rightarrow X$ be the universal covering of $X$.

Definition 1. Let $M$ be a complex manifold of dimension $n$ and let $L$ be a line bundle on $M . \quad L$ is said to be big, if

$$
\limsup _{m \rightarrow+\infty} m^{-n} \operatorname{dim} H^{0}\left(M, \mathcal{O}_{M}\left(L^{\otimes m}\right)\right)>0
$$

holds

LEMMA 1 (Kodaira's lemma) ([5, Appendix]). Let $M$ be a smooth projective variety and let $D$ be a big divisor on $X$. Then there exists an effective $\boldsymbol{Q}$-divisor $E$ on $X$ such that $D-E$ is an ample $\boldsymbol{Q}$-divisor.

Since $K_{X}$ is big, there exists an effective $\boldsymbol{Q}$-divisor $E$ such that $K_{X}-E$ is ample. Let $E=\sum_{\imath=1}^{k} a_{i} E_{\imath}$ be the irreducible decomposition of $E$. Let $\sigma_{i} \in H^{0}\left(X, \mathcal{O}_{X}\left(E_{\imath}\right)\right)$ be a holomorphic section such that $\left(\sigma_{\imath}\right)=E_{\imath}$. Then by Kodaira's lemma there exists $C^{\infty}$ hermitian metrics $h_{0}$ on $K_{X}$ and $h_{\imath}$ on $\mathcal{O}_{X}\left(E_{\imath}\right)$ $(1 \leqq i \leqq k)$ respectively such that

$$
\omega_{X}=\operatorname{curv} h_{0}-\sum_{\imath=1}^{k} a_{\imath} \operatorname{curv} h_{\imath}
$$

is a Kähler form on $X$, where curv denotes $\sqrt{-1} \partial \partial \log$ (operator which takes the curvature form of a hermitian metric).

Definition 2. Let $M$ be a complex manifold and let $L$ be a holomorphic line bundle on $M . \quad h$ is said to be a singular hermitian metric on $L$ if there exists a $C^{\infty}$ hermitian metric $h_{0}$ on $L$ and a locally $L^{1}$ function $\varphi$ such that

$$
h=e^{-\varphi} h_{0}
$$

holds. We define the curvature current curv $h$ by

$$
\operatorname{curv} h=\operatorname{curv} h_{0}+\sqrt{-1} \partial \bar{\partial} \varphi
$$

where curv $h_{0}=\sqrt{-1} \bar{\partial} \partial \log h_{0}$ is the usual curvature form and $\partial \bar{\partial}$ of $\varphi$ is taken in the sense of current.

DEFINITION 3. Let $T$ be a closed positive $(1,1)$ current on a complex manifold $M . T$ is said to be strictly positive, if for every point $x \in M$, there exists an open neighborhood $U_{x}$ and a $C^{\infty}$ Kähler form $\omega_{x}$ such that $T-\omega_{x}$ is a closed positive current on $U_{x}$.

We set

Let $\sigma_{\imath}$ be a holomorphic section of $\mathcal{O}_{X}\left(E_{\imath}\right)$ with divisor $E_{\imath}$ respectively. 


$$
h=\frac{h_{0}}{\prod_{\imath=1}^{k} h_{i}\left(\sigma_{\imath}, \sigma_{\imath}\right)^{a}} .
$$

$h$ is a singular hermitian metric on $X$ and satisfies

$$
\operatorname{curv} h=\omega+\sum_{\imath=1}^{k} a_{\imath} E_{\imath} .
$$

In particular $h$ has strictly positive curvature current. Then $\pi_{X}^{*} h$ is a singular hermitian metric of $K_{D}$ with strictly positive curvature current. We denote $\pi^{*} \omega_{X}$ by $\omega$ and $\pi_{X}^{*} h$ again by $h$ for simplicity. The following theorem follows from the standard $L^{2}$-estimate for $\bar{\partial}$-operator due to Hörmander.

THEOREM 2 ([6, p. 561]). Let $\left(M, \omega_{M}\right)$ be a complete Kähler manifold and let $\left(L, h_{L}\right)$ be a singular hermitian line bundle on $M$ such that

$$
\operatorname{curv} h_{L}+\operatorname{Ric}_{M} \geqq c \omega_{M}
$$

holds for some positive constant c. Let $\mathcal{L}^{2}\left(L, h_{L}\right)$ denote the sheaf of germs of local $L^{2}$ holomorphic sections of $\left(L, h_{L}\right)$. Then we have

$$
H_{(2)}^{q}\left(M, \mathcal{L}^{2}\left(L, h_{L}\right)\right)=0
$$

holds for every $q \geqq 1$ and $\mathcal{L}^{2}\left(L, h_{L}\right)$ is a coherent sheaf of $\mathcal{O}_{X}$-module.

\section{CORORALLY 1.}

$$
H_{(2)}^{0}\left(D, \mathcal{L}^{2}\left(K_{D}^{\otimes m}, h^{\otimes m}\right)\right) \longrightarrow \mathcal{L}^{2}\left(K_{D}^{\otimes m}, h^{\otimes m}\right) / \mathscr{M}_{x} \cdot \mathscr{M}_{y}
$$

is surjective for every $x, y \in D$, where $\mathscr{M}_{x}$ (resp. $\mathscr{M}_{y}$ ) denotes the maximal ideal sheaf at $x$ (resp. $y$ ).

Proof. The following proof is routine. First we shall consider the case $x \neq y$. Let $r_{x}$ (resp. $r_{y}$ ) denote the distance function from $x$ (resp. $y$ ) with respect to the Kähler form $\omega$. And let $U_{x}$ (resp. $U_{y}$ ) be a small open neighbourhoods of $x$ (resp. $y$ ) let $W_{x}$ (resp. $W_{y}$ ) be an open neighbourhood of $x$ (resp. y) such that $W_{x} \Subset U_{x}$ (resp. $W_{y} \Subset U_{y}$ ). Let $\rho$ be a nonnegative $C^{\infty}$ function such that $\rho \equiv 1$ on $W_{x} \cup W_{y}$ and Supp $\rho \subset U_{x} \cup U_{y}$. We set $\phi=$ $(2 n+2) \rho\left(\log r_{x}+\log r_{y}\right)$. Noting that $\phi$ is plurisubharmonic on a neighbourhood of $x$ and $y$, by direct calculation we see that there exists a positive constant $c$ such that

$$
\sqrt{ }-1 \partial \bar{\partial} \phi>-c \omega
$$

holds on $D$, there exists a positive integer $m$ such that

$$
m \operatorname{curv} h+\sqrt{-1} \partial \bar{\partial} \log \phi+\operatorname{Ric}_{\omega} \geqq \omega
$$

holds on $D$. Then by the above vanishing theorem 


$$
H_{(2)}^{q}\left(D, \mathcal{L}^{2}\left(L^{\otimes m}, e^{-\psi} h^{\otimes m}\right)\right)=0
$$

holds for every $q \geqq 1$. Hence by the definition of $\psi$,

$$
H_{2}^{0}\left(D, \mathcal{L}^{2}\left(L^{\otimes m}, h^{\otimes m}\right)\right) \longrightarrow \mathcal{L}^{2}\left(L^{\otimes m}, h^{\otimes m}\right) / \mathscr{M}_{x} \cdot \mathscr{M}_{y}
$$

is surjective. In the case of $x=y$, the proof is similar.

Q.E.D. of $D$.

By Corollary $1, H_{(2)}^{0}\left(D, \mathcal{L}^{2}\left(K_{D}^{\otimes^{m}}, h^{\otimes m}\right)\right)$ separates generates general points

Let $\Gamma$ be a discrete cocompact subgroup of $\operatorname{Aut}(D)$ acting $D$ without fixed point. Let $\sigma$ be a nontrivial $L^{2}$ holomorphic section of $K_{D}^{\otimes m}$. For $k \geqq 2$, we set

$$
a v\left(\sigma^{\otimes k}\right)=\sum_{\gamma \in \Gamma} \gamma^{*} \sigma^{\otimes k} .
$$

At this moment, $a v\left(\sigma^{\otimes k}\right)$ is not well defined because $\Gamma$ may not be an isometry.

To show that $a v\left(\sigma^{\otimes k}\right)$ is well defined, we shall use the measure hyperbolicity of $D$. We shall review the definition of measure hyperbolic manifolds. Let $M$ be an $n$-dimensional connected complex manifold. Let $\Delta^{n}$ denote the unit open polydisk in $C^{n}$. Let us take a point $x \in M$. Let $f: \Delta^{n} \rightarrow M$ be a holomorphic mapping such that $f(O)=x$ and $f$ is nondegenerate at 0 . Let $\Omega_{0}$ be the Poincaré volume form on $\Delta^{n}$ defined by

$$
\Omega_{0}=\prod_{\imath=1}^{n} \frac{4}{\left|z_{\imath}\right|^{2}\left(\log \left|z_{\imath}\right|\right)^{2}}(\sqrt{-1})^{n} d z_{1} \wedge d \bar{z}_{1} \wedge \cdots \wedge d z_{n} \wedge d \bar{z}_{n} .
$$

By inverse function theorem there exists $0<r<1$ and a neighbourhood $U$ of $x=f(0)$ such that $f \mid \Delta^{n}(r): \Delta(r)^{n} \rightarrow U$ is a biholomorphic mapping, where $\Delta^{n}(r)$ denotes the polydisk of radius $r$ with center $O$. We set

and

$$
\Psi_{M, f}(x)=\left(f^{-1} \mid \Delta(r)^{n}\right) * \Omega_{0}(O)
$$

$$
\Psi_{M}(x)=\inf \left\{\Psi_{M, f}(x)\right\},
$$

where the infimum is taken for all holomorphic mapping $f: \Delta^{n} \rightarrow M$ such that $f(O)=x$ and nondegenerate at $O$. Then $\Psi_{M}$ is a pseudo-volume form on $M$. We call $\Psi_{M}$ the hyperbolic volume form of $M$. It is easy to check that $\Psi_{M}$ is an upper semicontinuous $2 n$-form on $M$. $\Psi_{M}$ defines a measure $\mu_{M}$ on $M$. We call the measure $\mu_{M}$ the hyperbolic measure of $M$. $M$ is said to be measure hyperbolic, if $\mu_{M}(B)>0$ for any non-empty open subset $B \subset M$. The following propositions are well known (cf. [3]).

PROPOSITION 1. Let $M$ be a projective manifold of general type. Then $M$ is measure hyperbolic.

Proposition 2. Let $M$ be a complex manifold and let $\pi: \tilde{M} \rightarrow M$ be an unramified covering. Then $\Psi_{\tilde{M}}=\pi * \Psi_{M}$ holds. In particular $M$ is measure hyperbolic if and only if $\tilde{M}$ is measure. 
Using Proposition 1, 2, we see that $D$ is measure hyperbolic and $\Gamma$ is measure preserving with respect to the hyperbolic measure $\mu_{D}$.

LEMMA 2. There exists a constant $C>1$ such that

$$
\frac{1}{C} \int_{D}\|f\|^{2} \mu_{D} \leqq \int_{D}\|f\|^{2} \omega^{n} \leqq C \int_{D}\|f\|^{2} d \mu_{D}
$$

holds for every $f \in H_{(2)}^{0}\left(D, \mathcal{O}_{D}\left(K_{D}^{\otimes^{m}}\right)\right)$.

Proof. Let $F$ be a fundamental domain of $\pi_{1}(X)$. Since $\mu_{D}$ and $\omega$ are $\pi_{1}(X)$-invariant, it is sufficient to prove that there exist positive constants $C_{1}$, $C_{2}$ such that for every $f \in H^{0}\left(\bar{F}, \mathcal{O}_{D}\left(K_{D}^{\otimes m}\right)\right.$ ) (where $\bar{F}$ denote the closure of $F$ ),

$$
\int_{F}\|f\|^{2} \mu_{D} \leqq C_{1} \int_{F}\|f\|^{2} \omega^{n}
$$

and

$$
\int_{F}\|f\|^{2} \omega^{n} \leqq C_{2} \int_{F}\|f\|^{2} d \mu_{D}
$$

hold. Since $\Psi_{D}$ is $\pi_{1}(X)$-invariant, $\Psi_{D} / \omega^{n}$ is bounded from above by the definition of $\Psi_{D}$, this implies the existence of $C_{1}$.

Suppose that $C_{2}$ does not exist. Then there exists a sequence $\left\{f_{j}\right\}_{j=1}^{\infty}, f_{j} \in$ $H^{0}\left(\bar{F}, \mathcal{O}_{D}\left(K_{D}^{\otimes m}\right)\right)$ such that

$$
\int_{F}\left\|f_{j}\right\|^{2} \omega^{n}=1
$$

and

$$
\int_{F}\left\|f_{j}\right\|^{2} d \mu_{D} \leqq \frac{1}{2^{j}}
$$

By the plurisubharmonicity of the square of the absolute value of a holomorphic function, we see that for every relatively compact subset $W$ of $F$, there exists a constant $C_{W}$ such that

$$
\|f\|^{2}(x) \leqq C_{W} \int_{F}\|f\|^{2} \omega^{n}
$$

holds for every $x \in W$ and $f \in H^{0}\left(F, \mathcal{O}_{D}\left(K_{D}^{\otimes m}\right)\right)$. Since $\Psi_{D}$ vanishes only on some measure 0 subset of $D$, this is a contradiction.

Q.E.D.

LEMMA 3. There exists a positive constant $c$ such that

holds.

$$
h \cdot \Psi_{D}>c
$$

Proof. We note that $h$ and $d \mu_{D}$ are both $\pi_{1}(X)$ invariant. Hence we can identify $h^{-1}$ and $d \mu_{D}$ volume forms on $X$. Let $f: \Delta^{n} \rightarrow X$ be a holomorphic mapping. Then since curv $h$ a Kähler form on $X$, by the maximal principle (Schwarz lemma) there exists a positive constant $c$ independent of $f$ such that 


$$
f * h^{-1} \leqq c \Omega_{0}
$$

holds. Then by the definition of $d \mu_{D}$, we completes the proof of Lemma 2 .

Q.E.D.

We note that $\Psi_{D}^{-1}$ is a $\Gamma$-invariant singular hermitian metric of the canonical bundle $K_{D}$. By Lemma 2 and Lemma 3, we see that $a v\left(\sigma^{\otimes k}\right)$ is well defined for every $k \geqq 2$. We set

$$
\varphi=\frac{a v\left(\sigma^{\otimes 2 k}\right)}{a v\left(\sigma^{\otimes 2}\right)^{\otimes k}} .
$$

$\varphi$ is well defined by the following lemma.

LEMMA 4. We may assume that av( $\left.\boldsymbol{\sigma}^{\otimes 2}\right)$ is not identically zero. We set

Proof. Let $x$ be a point on $D$. Let $\eta$ be a generator of $K_{D}^{\otimes m}$ around $x$.

$$
a_{\gamma}=\frac{r^{*} \sigma}{\eta}
$$

Suppose that $a v\left(\sigma^{\otimes 2 k}\right) \equiv 0$ for every $k \geqq 1$. Then

$$
\sum_{\gamma \in \Gamma} a_{r}^{2 k} \equiv 0
$$

holds around $x$ for every $k$. But this implies that $a_{\gamma} \equiv 0$ around $x$ for every $\gamma$. This is the contradiction. Hence replacing $\sigma$ by $\sigma^{\otimes l}$ for some $l$, if necessary, we may assume that $a v\left(\sigma^{\otimes 2}\right)$ is not identically 0 .

Q.E.D.

LEMMA 5. $\varphi$ is a nonconstant $\Gamma$-invariant meromorphic function for some $k$.

Proof. Suppose that $\varphi$ is constant for every $k$. Let $x$ be a point on $D$ such that $a v\left(\sigma^{\otimes 2}\right)(x) \neq 0$. Let $y$ be a point on $D$. We set

$$
f_{r}=\frac{\gamma^{*} \sigma^{\otimes 2}}{a v\left(\sigma^{\otimes 2}\right)} .
$$

Since $\varphi$ is constant for every $k$,

$$
\sum_{\gamma \in \Gamma} f_{r}(x)^{k}=\sum_{\gamma \in \Gamma} f_{r}(y)^{k}
$$

holds for every $k$. This implies that

$$
\left\{f_{\gamma}(x)\right\}=\left\{f_{\gamma}(y)\right\}
$$

holds. Hence by moving $y$ on a neighbourhood of $x$, we see that $f_{r}$ is constant. Since $a v\left(\sigma^{\otimes 2}\right)$ is $\Gamma$ invariant and $D$ is noncompact, this contradicts the fact that $\sigma$ is a $L^{2}$-holomorphic section.

Q.E.D. 
Let $K$ be the function field generated by such $\varphi$. Then there exists a projective variety $B$ whose function field corresponds $K$. Let $r: \Gamma \backslash D-\cdots \rightarrow B$ be a rational map induced by the inclusion $K(B) \subset K(I \backslash D)$. Let $\mu:(\Gamma \backslash \sim D) \rightarrow \Gamma \backslash D$ be a resolution of the base locus of $r: \Gamma \backslash D-\cdots \rightarrow B$. Let $\tilde{r}: \Gamma \backslash \sim D \rightarrow B$ be the natural morphism. Let $\tilde{Y}$ be a general fibre of $\tilde{r}$ and let $Y$ denote $\mu(Y)$. Suppose that $\operatorname{dim} Y \geqq 1$. Let $\pi_{\Gamma}: D \rightarrow \Gamma \backslash D$ be the natural projection. Then $\pi_{\Gamma}^{-1}(Y)$ is a $\Gamma$ invariant subvariety of $D$ and every element of $K$ is constant on $\pi_{\Gamma}^{-1}(Y)$. We note that $H_{(2)}^{0}\left(D, \mathcal{L}^{2}\left(K_{D}^{\otimes m}, h^{\otimes m}\right)\right)$ separates general point of $\pi_{\Gamma}^{-1}(Y)$, if we take $Y$ sufficiently general. Using this fact, repeating the same argument as above, we can construct an element of $K$ which is nonconstant on $\pi_{\Gamma}^{-1}(Y)$. This is the constradiction.

In conclusion, $K$ separates the general points of $\Gamma \backslash D$. Hence $\Gamma \backslash D$ is of general type. This completes the proof of Theorem 1 .

\section{REFERENCES}

[1] T. Aubin, Equation du type Monge-Ampère sur les variété Kähl ériennes compactes, C.R. Acad. Sci. Paris, 283 (1976), 119-121.

[2] M. Gromov, Kähler hyperbolicity and $L^{2}$-Hodge theory, J. Differential Geom., 33 (1991), 263-292.

[3] S. Kobayashi, Hyperbolic Manifolds and Meromorphic Mappings, Dekker, New York, 1970.

[4] S. Kobayashi And T. OchiaI, Meromorphic mappings onto compact complex spaces of general type, Invent. Math., 31 (1975), 7-16.

[5] S. Kobayashi And T. Ochial, Mappings into compact complex manifolds with negative first Chern class, J. Math. Soc. Japan, 23 (1971), 137-148.

[6] A.M. NADEL, Multiplier ideal sheaves and existence of Kähler-Einstem metrics of positive scaler curvature, Ann. of Math., 132 (1990), 546-596.

[7] S.-T. YAU, On the Ricci curvature of compact Kähler manifold and the complex Monge-Ampére equation I, Comm. Pure Appl. Math., 31 (1978), 339-411.

[8] S.-T. YAU, A general Schwarz lemma for Kähler manifolds, Amer. J. Math., 100 (1978), 197-203.

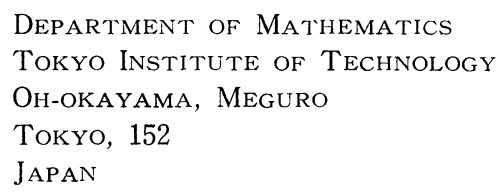


